\title{
Temporalité et attitude : structuration du discours et expression de la modalité, Arie Molendijk et Co Vet
}

\section{Luigi Luison}

\section{Q OpenEdition}

1 Journals

\section{Edizione digitale}

URL: http://journals.openedition.org/studifrancesi/31041

DOI: 10.4000/studifrancesi.31041

ISSN: 2421-5856

\section{Editore}

Rosenberg \& Sellier

\section{Edizione cartacea}

Data di pubblicazione: 1 avril 2006

ISSN: 0039-2944

\section{Notizia bibliografica digitale}

Luigi Luison, «Temporalité et attitude : structuration du discours et expression de la modalité, Arie Molendijk et Co Vet», Studi Francesi [Online], 148 (XLX | I) | 2006, online dal 30 novembre 2015, consultato il 18 avril 2021. URL: http://journals.openedition.org/studifrancesi/31041 ; DOI: https:// doi.org/10.4000/studifrancesi.31041

Questo documento è stato generato automaticamente il 18 avril 2021.

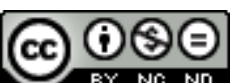

Studi Francesi è distribuita con Licenza Creative Commons Attribuzione - Non commerciale - Non opere derivate 4.0 Internazionale. 


\title{
Temporalité et attitude : structuration du discours et expression de la modalité, Arie Molendijk et Co Vet
}

\author{
Luigi Luison
}

\section{NOTIZIA}

AA.VV., Temporalité et attitude : structuration du discours et expression de la modalité,

“Cahiers Chronos" 12. Textes réunis par Arie MOLENDIJK et Co VET, Amsterdam, Èditions

Rodopi, 2005, pp. 231.

1 Il presente volume è il frutto del $\mathrm{V}$ colloquio Chronos che si è tenuto all'Università di Groningen dal 19 al 21 giugno 2002. In tale sede sono state affrontate tre grandi tematiche di ordine linguistico che concernono le varie lingue: la struttura del discorso, l'espressione di modalità differenti, la temporalità e l'aspetto.

2 André BORILLo (Les adverbes temporels et la structuration du discours, p. 1-18) apre la raccolta illustrando lo sviluppo del ruolo di alcuni avverbi temporali che fungono da connettori nella strutturazione del discorso, in particolare in quello narrativo. Questi avverbi contribuiscono alla sua coerenza, stabilendo tra le frasi alcune relazioni argomentative, secondo il modello dei connettori discorsivi.

3 Anne LE DRAOULEC (Connecteurs temporels d'immédiateté: le cas de "aussitôt " et "soudain", pp. 19-34), rimanendo nell'ambito dei connettori discorsivi, entra più nello specifico studiando lo statuto di connettore di due avverbi aspetto-temporali, aussitôt e soudain, nelle sequenze frasali al passato prossimo.

4 Jacques MOESCHLER (Connecteurs pragmatiques, inférences directionnelles et représentation mentales, pp. 35-50) parte dalla teoria della Pertinenza per sostenere la tesi secondo cui i connettori pragmatici devono essere trattati come espressioni procedurali. L'attenzione dell'A. si focalizza soprattutto sui connettori impiegati nelle inferenze 
temporali e causali (et e parce que) e spiega qual è il loro contributo per la comprensione del discorso.

Patrick CAUDAL e Laurent ROUSSARIE (Sémantique et pragmatique des propositions en " si", pp. 51-66) propongono un utilizzo unificato dei principali usi delle preposizioni con si. Il quadro teorico nel quale gli autori situano le loro ricerche è quello delle recenti versioni della SDRT (Segmented Discourse Representation Theory).

Alexander LOENGARov ("le fait que..." et la question du subjonctif: la directionnalité de la grammaticalisation, pp. 67-81) si pone l'obiettivo di studiare l'impiego modale dopo il sintagma "le fait que...", verificando, da una parte, in che modo l'opposizione tra indicativo e congiuntivo dopo "le fait que..." è rapportato all'uso esclusivo di uno dei due modi, secondo la teoria della grammaticalizzazione; dall'altra, verificando in che modo il francese permette di sfruttare l'alternanza modale tra indicativo e congiuntivo nella sfera semantica.

7 Jean-Claude SOUESME (Questions modalisées et intonation, pp. 83-96), nell'ambito delle operazioni enunciative, analizza i fenomeni intonativi in funzione di criteri linguistici nelle frasi interrogative in lingua inglese con verbi modali.

Merete BIRKELUND (Négation et modalité, pp. 97-108) presenta un doppio lavoro di ricerca: interpretare la scelta operata dal locutore per esprimere gli obblighi contrattuali e analizzare l'uso della negazione ne...pas negli enunciati con i verbi modali devoir e pouvoir, proponendo, nel contempo, una spiegazione del perché il locutore di testi contrattuali preferisca usare il presente, il futuro semplice e il verbo pouvoir a detrimento del verbo modale devoir.

Patrick CAUDAL e Carl VETTERS (Un traitement conjoint $d u$ conditionnel, $d u$ futur et de l'imparfait: les temps comme des fonctions d'acte de langage, pp. 109-124) si soffermano sull'argomento centrale di questa raccolta, quello aspetto-temporale. Gli autori, proponendo un trattamento congiunto del condizionale, del futuro e dell'imperfetto, cercano di dimostrare che il condizionale associa la semantica dell'imperfetto in quanto indicatore del non attualizzato e quella del futuro in quanto indicatore di una consecuzione.

Véronique LAGAÉ (Les formes en "être " participe passé à valeur résultative dans le système verbal français, pp. 125-142) parte dall'analisi di due frasi tipo come le cadeau est déjà emballé e Pierre est parti depuis une heure, per dimostrare come sia difficoltoso stabilire, dal punto di vista semantico, un legame tra la forma con il verbo être + participe passé e un'altra forma, dal momento che, se la costruzione con être + participe passé viene considerata una costruzione attributiva, si esclude l'impiego di altre possibilità linguistiche, come ad esempio quella di usare questa forma in una costruzione impersonale.

11 Greta комUR (Le transfert de l'aspect verbal sur le substantif en polonais, pp. 143-157) apporta il suo contributo con l'analisi di sostantivi polacchi che derivano da un verbo. I sostantivi verbali che l'A. prende in esame possiedono una funzione simile a quella dell'infinito, ma vengono indeboliti da veri sostantivi. Nell'ambito dei sostantivi verbali è opportuno, secondo l'A., effettuare una distinzione tra nomi astratti e nomi concreti. Le argomentazioni dell'A. tendono a dimostrare che solo i nomi astratti veicolano l'opposizione perfettivo/ imperfettivo in un modo simile agli infiniti.

12 Maria ANTONIOU (Le passé composé du français: une forme verbale à valeur temporelle?, pp. 159-170) mette in evidenza che il passé composé non è una forma utile a localizzare un 
processo nella forma al passato e che tutti i suoi usi possono essere spiegati in maniera univoca: il passé composé veicola invariabilmente la momentaneità.

Bissera IANKOVA-GORGATCHEV (Le "Present Perfect" résultatif: marqueurs aspectuels, pp. 171-185) affronta il problema del tempo e dell'aspetto a partire dall'analisi delle forme verbali in funzione dei loro contesti. Lo studio svolto dall'A. verte su un corpus di quattrocento enunciati che implicano il Present Perfect.

PAUL-LOUIS THOMAS (Recomposition du système aspecto-temporel en serbo-croate: bosniaque, croate, monténégrin, serbe, pp. 187-201) presenta un lavoro sull'uso delle forme verbali del passato nelle traduzioni moderne in serbo-croato di alcuni estratti del Nuovo Testamento.

EMMANUELle LABEAU (Des "temps modernes" : l'aspect suffit-il à la maîtrise des temps du passé dans les narrations écrites d'apprenants avancés?, pp. 203-218) esamina la teoria "Primacy of Aspect Hypothesis" di Robinson e quella "Lexical Aspect Hypothesis" di Andersen, secondo le quali, nell'interlingua di coloro che apprendono una lingua straniera, $i$ morfemi verbali della lingua d'arrivo sono utilizzati per indicare l'aspetto lessicale, indipendentemente dalla loro funzione con un uso alla forma negativa.

DANY AMIOT, WALTER DE MULDER e Nelly FLAUX (Nous sommes dimanche, pp. 219-231) chiudono questa raccolta con l'analisi della forma temporale nous sommes dimanche. Gli autori cercano di dare delle risposte a tre interrogativi fondamentali: perché è possibile escludere tutti gli altri pronomi personali definiti tranne nous, quale tipologia d'uso entra in gioco usando il verbo être, qual è lo statuto semantico-sintattico della parola dimanche. 